\title{
Can We STOPP Falls?
}

\author{
(D) Nathalie van der Velde, (D) Lotta Seppala \\ Amsterdam University Medical Centers, Amsterdam, Netherlands
}

\section{Keypoints}

- Falls are under-recognized as adverse drug events (ADEs),

- Healthcare professionals are reluctant to deprescribe fall-risk increasing drugs,

- A European expert consensus list of fall-risk increasing drugs was developed to aid healthcare professionals in identifying fall-risk increasing drugs,

- The STOPP falls deprescribing instrument was developed to assist health care professionals to assist clinical decision making and facilitate appropriate deprescribing of fall-risk increasing drugs,

- STOPP falls is more comprehensive than most national falls prevention guideline listings,

- STOPP falls is a first step toward harmonizing practice and guidelines on drug-related falls in Europe.

Occurrence of harm from medication use is rapidly increasing, especially in older persons, and so is harm resulting from fall incidents in this age group $(1,2)$. Although at first sight these two rising health care problems appear to be separate harms, they are in fact strongly interrelated. In the majority of cases, falls result from interacting risks; and certain medication classes are a significant risk factor for falls (3-5). Thus, falls, widely acknowledged as a geriatric syndrome, should also be recognized as a commonly occurring ADE in the older population (6). Given the fact that approximately 0.9 to $1.5 \%$ of the total health care budget in Western countries is spent on fall-related costs (7), there is a great need to prevent unnecessary falls and related injury. This includes minimization of the ADE "falls".

Since the use of fall-risk-increasing drugs (FRIDs) is one of the most prominent fall risk factors, a medication review -aimed at deprescribing FRIDs- is considered an essential component of the multifactorial falls intervention in (inter)national falls prevention guidelines $(8,9)$. In general it is acknowledged that the major groups of medications that can increase fall risk are psychotropic drugs and cardiovascular drugs. Three recent systematic reviews and meta-analyses confirmed the association between psychotropics [antidepressants (selective serotonin reuptake inhibitors, tricyclic antidepressants), antipsychotics, benzodiazepines] and fall risk (4). Moreover, consistent associations were reported for loop diuretics, antiepileptics, opioids, and polypharmacy $(3,5)$. Digitalis, nonselective beta-blocking agents, antiarrhythmics, diuretics in general, antihypertensives, anticholinergics, non-steroidal anti-inflammatory drugs, analgesics, laxatives, long-term proton pump inhibitors, and antiplatelets were also identified as possible FRIDs $(3,5)$. However, although FRIDs use is common in older persons, health care professionals are often reluctant to deprescribe FRIDs (6). Knowledge is lacking among both health care professionals and older people and their caregivers concerning the role of medication as a fall risk factor. The members of the European Geriatric Medicine Society (EuGMS) task and finish group on FRIDs have taken it upon themselves to facilitate appropriate (de)prescribing in older persons at risk of falls through raising public awareness, knowledge dissemination activities, update of knowledge, development of personalized and effective deprescribing interventions, all aimed at optimizing and harmonizing practices among Europe (10). The group was founded by us in 2016 and by now includes 30 members from 14 European countries. On the website of the EuGMS all publications, developed materials as well as symposium presentations can be found and downloaded.

The main objectives of the T\&tF group on FRIDs are:

- To update knowledge related to FRIDs. 
- To successfully disseminate knowledge on FRIDs and FRIDs withdrawal to health care workers, students and the older population at risk and thus lessen the use of unnecessary FRIDs in older persons at risk.

- To develop drug withdrawal interventions that are personalized and effective.

- To harmonize practice on this topic across Europe.

The latest accomplishment of our group, in collaboration with the EuGMS SIG on pharmacology, is the development of the STOPP falls deprescribing instrument, which was recently published in age and ageing (11). The tool provides practical guidance to simplify and structure FRIDs deprescribing in clinical practice and it has been translated to a freely available online available digital deprescribing decision support tool (STOPP falls online tool).

STOPP falls was built through a Delphi process of European experts and resulted in an agreed on list of 14 FRIDs, in majority psychotropic medication and furthermore cardiovascular and other drug groups such as anticholinergics (11). Also 18 differences between pharmacological subclasses were identified with regard to fall-risk-increasing properties and practical deprescribing guidance to assist clinical decision making. This information was summarized in overview tables and made easily interpretable by providing decision trees per medication group. Remarkably, the STOPP fall list is more comprehensive than most national falls prevention guideline listings.

The drug groups that consensus was reached on as being fallrisk increasing are:

- Benzodiazepines and benzodiazepine related drugs,

- Antipsychotics,

- Antidepressants,

- Diuretics,

- Alpha-blockers used as antihypertensives,

- Centrally-acting antihypertensives,

- Vasodilators used in cardiac diseases,

- Opioids,

- Antiepileptics,

- Anticholinergics,

- Alpha-blockers used for prostate hyperplasia,

- Overactive bladder and incontinence medications,

- Sedative antihistamines.

In the final consensus step of the Delphi effort, consensus was reached on guidance advice of deprescribing, which was translated to a stepwise deprescribing tool, that can also be found online (12). The deprescribing guidance includes information on when and how to deprescribe with relevant references and links to general deprescribing guidelines, specifically is addressed:

- In which cases to consider deprescribing (symptoms and/or indications),

- If stepwise withdrawal is needed,

- If monitoring after deprescribing is necessary (including symptoms and frequency).

STOPP falls is formally part of the STOPP/START series and the results will be included in the draft criteria for the anticipated STOPP/START version 3, to be further validated by the STOPP/ START panelists. A next step of our group will be to obtain funding to validate the tool and assess its effectiveness on falls prevention, preferably in a European, multicenter and multicountry randomized controlled study. But in between we are performing a systematic review and meta-analysis to summarize evidence on the effectiveness of different deprescribing tools (including general ones) on fall risk and related injury. This paper is anticipated to be published second part of 2021 and will inform the recommendations of the ongoing word falls guideline effort (13).

Keywords: Deprescribing, older people, falls, prevention, medication review

\section{Ethics}

Peer-review: Internally peer-reviewed.

\section{Authorship Contributions}

Concept: N.V.D.V., L.S., Design: N.V.D.V., L.S., Literature Search: N.V.D.V., L.S., Writing: N.V.D.V., L.S.

Conflict of Interest: No conflict of interest was declared by the authors.

Financial Disclosure: The authors declared that this study received no financial support.

\section{References}

1. Veeren JC, Weiss M. Trends in emergency hospital admissions in England due to adverse drug reactions: 2008-2015. J Pharm Health Serv Res 2017;8:511.

2. Haagsma JA, Olij BF, Majdan M, van Beeck EF, Vos T, Castle CD, Dingels ZV, Fox JT, Hamilton EB, Liu Z, Roberts NLS, Sylte DO, Aremu O, Bärnighausen TW, Borzi AM, Briggs AM, Carrero JJ, Cooper C, El-Khatib Z, Ellingsen $C L$, Fereshtehnejad SM, Filip I, Fischer F, Haro JM, Jonas JB, Kiadaliri AA, Koyanagi A, Lunevicius R, Meretoja TJ, Mohammed S, Pathak A, Radfar A, Rawaf S, Rawaf DL, Riera LS, Shiue I, Vasankari TJ, James SL, Polinder S. Falls in older aged adults in 22 European countries: incidence, mortality and burden of disease from 1990 to 2017. Inj Prev 2020;26(Supp 1):i67-i74.

3. de Vries M, Seppala L, Daams JG, van de Glind EMM, Masud T, van der Velde N; EUGMS Task and Finish Group on Fall-Risk-Increasing Drugs. Fall-Risk- 
Increasing Drugs: A Systematic Review and Meta-Analysis: I. Cardiovascular Drugs. J Am Med Dir Assoc 2018;19:371.e1-371.e9.

4. Seppala $\sqcup$, Wermelink AMAT, de Vries M, Ploegmakers KJ, van de Glind EMM, Daams JG, van der Velde N; EUGMS task and Finish group on fallrisk-increasing drugs. Fall-Risk-Increasing Drugs: A Systematic Review and Meta-Analysis: II. Psychotropics. J Am Med Dir Assoc 2018;19:371.e11-371. e17.

5. Seppala $\amalg$, van de Glind EMM, Daams JG, Ploegmakers KJ, de Vries M, Wermelink AMAT, van der Velde N; EUGMS Task and Finish Group on FallRisk-Increasing Drugs. Fall-Risk-Increasing Drugs: A Systematic Review and Meta-analysis: III. Others. J Am Med Dir Assoc 2018;19:372.e1-372.e8.

6. Seppala $U$, van der Velde $N$, Masud $T$, Blain $H$, Petrovic $M$, van der Cammen TJ, Szczerbinska K, Hartikainen S, Kenny RA, Ryg J. EuGMS Task and Finish group on Fall-Risk-Increasing Drugs (FRIDs): Position on Knowledge Dissemination, Management, and Future Research. Drugs Aging 2019;36:299-307.

7. Centers for Disease Control and Prevention. Injury Prevention \& Control. Keep on your feet - Preventing Older Adult Falls. https://www.cdc.gov/ injury/features/older-adult-falls/index.html. Accessed April 20th 2020.
8. Gillespie LD, Robertson MC, Gillespie WJ, Sherrington C, Gates S, Clemson LM, Lamb SE. Interventions for preventing falls in older people living in the community. Cochrane Database Syst Rev 2012;2012:CD007146.

9. Panel on Prevention of Falls in Older Persons, American Geriatrics Society and British Geriatrics Society. Summary of the Updated American Geriatrics Society/British Geriatrics Society clinical practice guideline for prevention of falls in older persons. J Am Geriatr Soc 2011;59:148-157.

10. https://www.eugms.org/research-cooperation/task-finish-groups/frid-fallrisk-increasing-drugs.html

11. Seppala $\sqcup$, Petrovic M, Ryg J, Bahat G, Topinkova E, Szczerbińska K, van der Cammen TJM, Hartikainen S, Ilhan B, Landi F, Morrissey Y, Mair A, GutiérrezValencia $M$, Emmelot-Vonk MH, Mora MÁC, Denkinger $M$, Crome $P$, Jackson SHD, Correa-Pérez A, Knol W, Soulis G, Gudmundsson A, Ziere G, Wehling M, O'Mahony D, Cherubini A, van der Velde N. STOPPFall (Screening Tool of Older Persons Prescriptions in older adults with high fall risk): a Delphi study by the EuGMS Task and Finish Group on Fall-Risk-Increasing Drugs. Age Ageing 2021;50:1189-1199.

12. https://kik.amc.nl/falls/decision-tree/

13. https://worldfallsguidelines.com/ 